\title{
Detection and Determination of the Variation of the Speed of Time
}

\author{
Robert M. L. Baker', Bonnie Sue Baker², Fangyu Li ${ }^{3}$ \\ ${ }^{1}$ Gravwave LLC, Playa del Rey, USA \\ ${ }^{2}$ Transportation Sciences Corporation, Gravitational-Wave Department, Playa del Rey, USA \\ ${ }^{3}$ Department of Physics, Chongqing University, Chongqing, China \\ Email: drrobert@gravwave.com, bonniesuebaker@gmail.com, fangyuli@cqu.edu.cn
}

How to cite this paper: Baker, R.M.L., Baker, B.S. and Li, F.Y. (2021) Detection and Determination of the Variation of the Speed of Time. Journal of Modern Physics, 12, 761780

https://doi.org/10.4236/jmp.2021.126049

Received: March 2, 2021

Accepted: May 9, 2021

Published: May 12, 2021

Copyright (c) 2021 by author(s) and Scientific Research Publishing Inc. This work is licensed under the Creative Commons Attribution International License (CC BY 4.0).

http://creativecommons.org/licenses/by/4.0/

\begin{abstract}
Steadily increasing time is involved in most scientific analyses. Like other dimensions in spacetime we suggest that there can be a variation rate of time's progress or speed of time in the time dimension. We study speed-of-time variation observational data in three processes: muon decay, galaxy rotation (related to dark matter) and the separation speed of celestial objects as our Universe progresses (related to dark energy). Each of these processes will have an "observed value" of their time of completion $P_{o}$ from an observation of the process at time $t_{1}$ and an "expected value" $P_{e}$ of that time at time $t_{2}$. Their difference is attributed to the variation of the speed of time. We provide a possible explanation for the anomalous separation of the observed and the expected galactic velocity curves. Our conclusion is that it is unnecessary to introduce dark matter or dark energy.
\end{abstract}

\section{Keywords}

Early Universe, High-Frequency Gravitational Waves, High-Frequency Relic Gravitational Waves, Primordial Gravitational Waves, Cosmology, Speed of Time, Dark Matter, Dark Energy, Galactic Velocity Curves

\section{Introduction}

We believe that although time, as a steadily increasing independent variable, is involved in almost all scientific analyses, time's rate of change (speed of time) and the variation of that speed should also be involved. We suggest that time, like the motion of objects moving in the three space dimensions, can increase or decrease at a variable rate. Similar to the hands of a watch moving fast or slow, this change in the speed of time could be almost trivially small or very large. In 
the standard cosmological model, the early universe was very smooth (homogeneous), but we suggest that since the beginning of our Universe entropy's evolution should be inhomogeneous, that is the rate of entropy increase cannot be uniform. Because the direction of the time arrow depends on the "direction" of entropy increase, the speed of time should also depend on the "speed" of entropy increase. In this case, if the entropy of the whole Universe has been increasing and entropy's speed is slowing down, then the speed of time is also slowing down! Here we apply the speed-of-time concept to observational data concerning three different physical processes: muon decay time, the rotational speed of the observable portions of a galaxy (related to dark matter) and the separation speed of celestial-objects as our Universe progresses (related to dark energy).

We also suggest that the detection of High-Frequency Gravitational Waves (HFGWs) is an essential observational tool for examining the speed-of-time concept:

1) Unlike the low-frequency gravitational waves (e.g., the gravitational waves generated by the merger of black holes or neutron stars) HFGWs are generated less than a nanosecond after the beginning of our Universe. We believe these primordial or relic HFGWs were generated by processes occurring when the speed of time in our early Universe was extremely fast.

2) Today almost all mainstream cosmological inflation models expect that the upper limit of the frequencies of primordial HFGWs should be $\mathrm{GHz}$ or higher. This means that the period of the primordial HFGWs is about $10^{-9}$ seconds or less. That time may be about the time necessary to complete an oscillation or essentially the time to complete some activity or process in our early Universe.

3) We contend that primordial or relic HFGWs were propagated before our Universe became transparent to electromagnetic radiation. If such primordial HFGWs can be detected by the HFGW detector, discussed in connection with our analyses of Muon decay, then their observations may not only contain information on the speed of time, but information, gained by means of the analyses of the HFGW frequency spectrum produced by the processes themselves.

4) In the future detection of primordial high-frequency gravitational waves, it seems necessary to distinguish what is the increase of the wavelength of the primordial gravitational waves due to the possible expansion of the Universe (i.e., the decrease of the frequency), and what is the decrease of the frequency due to the decrease of the speed of time, as our Universe ages. This determination may not only be a challenge, but also an important opportunity in the study of cosmology.

5) The observed speed of the stars comprising the periphery of nearby galaxies can be overestimated. Such an overestimates may be caused by the Doppler observation of stars beyond in spacetime the galaxy being miss-associated to be an actual peripheral galactic star. Since a star beyond the nearby galaxy would be further from the Earth than the galaxy and closer to the beginning of our Universe, they would be in a spacetime region of higher time speed and therefore higher apparent star speed relative to the observer on our Earth. Thus we may be fooled into associating them with the galactic stars and thereby overestimating the observational average speed of the peripheral galactic stars. 
We will also discuss processes that do not depend internally on the three space dimensions and are independent of the time-varying flow of time in our Universe. We call them Non-Varying-Rate-of-Time (NVRT) processes and suggest muon decay as an example of a NVRT process.

Most processes depend upon various parameters and variables, such as $a, b$, c... and time, but here we single out time as the variable of interest. We propose that the best way to determine the speed of time is to compare the same physical process at two different times. Each process, $P$, will have an "observed value" of the process' time of completion, $P_{o}$, from an observation of the process at time $t_{1}$ and an "expected value" of $P_{e}$ as the process time is expected to be at another time $t_{2}$. Time $t_{2}$ is usually considered in this discussion to be a time in the past when the photons left the process $P$. Or in the case of muon decay, when the process time, $P_{0}$ was obtained and recorded in the past at a time $\mathrm{t}_{1}$. If time is not progressing steadily and uniformly, then we attribute any variation of the expected Process $P_{e}\left(t_{2}\right)$ time from the Process time we actually observe or actually record, $P_{o}\left(t_{1}\right)$, to a variation of the speed of time. The fundamental equation relating $P_{o}\left(t_{1}\right)$ and $P_{e}\left(t_{2}\right)$ to determine the variation of the speed of time, $V_{s t}$ is:

$$
V_{s t}=\left[P_{o}\left(t_{1}\right)-P_{e}\left(t_{2}\right)\right] /\left(t_{1}-t_{2}\right) .
$$

If the observed time for a single cycle or for the completion of a Process, $P_{o}$ is exactly the same as the expected time for such a process, $P_{e}$ then time running smoothly with no variation, in which case is the Variation of the Speed of time is zero.

The Processes of interest and our expectations for them are:

1) The expected duration of muon decay at $t_{2}, P_{e}\left(t_{2}\right)$, is equal to the last measured value of muon decay time in picoseconds, at $t_{1}$.

2) The inverse of the expected speed of a portion of the visible disk of a galaxy at $t_{2}, P_{e}\left(t_{2}\right)$, in seconds as based upon conventional Astrodynamics [1] [2].

3) The expected value of the speed of separation of a celestial object at $t_{2}$, $P_{e}\left(t_{2}\right)$, is established by a "proposed" expansion theory of our Universe, here taken to be that the separation speed should be the same everywhere in our Universe (also that the Hubble "constant" is approximately $70 \mathrm{~km} / \mathrm{s}$ per Mpc or $2 \times 10^{-18}$ [m/s per meter] or approximately $1 / 5 \times 10^{17}$ seconds) therefore we express the expected cosmic object's speed in fractions of the Hubble "constant" in seconds, to be equal everywhere in our Universe.

\section{Muon Decay Time to Measure the Variation of the Speed of Time}

The most accurate time measurements of Process time in a laboratory on Earth were found to be the decay time of Muon's as measured by atomic or nuclear clocks. Muons are produced when cosmic rays strike atomic nuclei of molecules in the air and quickly decay over a fixed time interval. Muons can also be produced in a two-step process at large research facilities. High energy protons $(>500 \mathrm{MeV})$ generated by a particle accelerator collide into a carbon or beryllium target and generate Muons. 
The earliest measurement of muon decay time that we found was made in 1946 of 2,330,000 ps [3]. A more accurate measurement of muon-decay time found was 2,202,000 picoseconds (ps) by Eckhause, et al. in 1963 as part of the Olive, Particle Data Group [4] findings. The most accurate muon-decay time found so far was made by Webber and a group called the MuLan Collaboration in 2011 of $2,196,980 \mathrm{ps}$ [5].

After further search of the literature a mysterious trend appeared: the duration of muon decay, which should be a constant, appears to shorten gradually, perhaps irregularly (including pauses and acceleration or lengthening), from 1946 to 2017 from very roughly 2.330 microseconds (1946) to very roughly $2.202 \mathrm{mi}-$ croseconds (1962-1963) by Lindy [6] and could be a basis for the detection and determination of the variation of the speed of time effect in laboratories on Earth independent of relativistic effects, that is if it is found that the shortening of muon decay time continues to be observed.

All of these observable data are exhibited in Table 1 and graphed in Figure 1.

The 1946 Conversi, et al. measurement's estimated error was so large as to be eliminated, except as Clive Woods suggested, “... that if outliers were eliminated, then any possible trend might be masked." Therefore, if we include the 1946 Conversi, et al. measurement, we take the decay-time difference between both

Table 1. Review of length of apparent muon decay time versus time.

\begin{tabular}{|c|c|c|c|c|}
\hline $\begin{array}{c}\text { Date of } \\
\text { Measurement }\end{array}$ & $\begin{array}{l}\text { Apparent Muon } \\
\text { Decay Time } \\
\text { (Picoseconds) }\end{array}$ & $\begin{array}{l}\text { Estimated Error } \\
\text { (Picoseconds) }\end{array}$ & $\begin{array}{l}\text { Muons at Rest or in } \\
\text { high-speed Cosmic-ray } \\
\text { generated Motion? }\end{array}$ & Reference \\
\hline 1946.0 & $2,330,000$ & $\pm 150,000$ & At Rest & $\begin{array}{c}\text { Conversi, Pancini, } \\
\text { Piccioni [3] }\end{array}$ \\
\hline 1962.0 & $2,203,000$ & \pm 4000 & At Rest & Lindy [6] \\
\hline 1963.0 & $2,202,000$ & \pm 3000 & At Rest & Eckhause, et al. [4] \\
\hline 1973.0 & $2,19 \underline{7,300}$ & \pm 300 & At Rest & Duclos in Olive. [4] \\
\hline 1974.0 & $2,19 \underline{7,110}$ & \pm 80 & At Rest & Balandin in Olive. [4] \\
\hline 1984.0 & $2,19 \underline{6,950}$ & \pm 60 & At Rest & Giovanetti in Olive. [4] \\
\hline 1984.0 & $2,19 \underline{7,078}$ & \pm 73 & At Rest & Bardin in Olive. [4] \\
\hline 2007.0 & $2,19 \underline{7,013}$ & \pm 21 & At Rest & Chitwood in Olive. [4] \\
\hline 2008.0 & $2,19 \underline{7,083}$ & \pm 32 & At Rest & Barczyk in Olive. [4] \\
\hline 2008.5 & $2,19 \underline{7,030}$ & \pm 40 & At Rest & Coan \& Ye in Olive [4] \\
\hline 2009.5 & $2,196,980.3$ & \pm 2.2 & At Rest & Webber/MuLan [5] \\
\hline 2013.0 & $2,19 \underline{6,980.3}$ & \pm 2 & $\begin{array}{c}\text { At Rest; a copy of } \\
2009.5 \text { measurement }\end{array}$ & Tischchenko [7] \\
\hline $2015.0^{2}$ & $2,110,000$ & $\pm 70,000$ & Fast, Cosmic Ray & Barazandeh [8] \\
\hline $2015.0^{2}$ & $2,165,000$ & $\pm 403,000$ & Fast, Cosmic Ray & Barazandeh [8] \\
\hline 2016.0 & $2,078,000$ & $\pm 11,000$ & At Rest & Physics OpenLab [9] \\
\hline 2017.0 & $2,080,000$ & $\pm 11,000$ & At Rest & Adams [10] \\
\hline
\end{tabular}




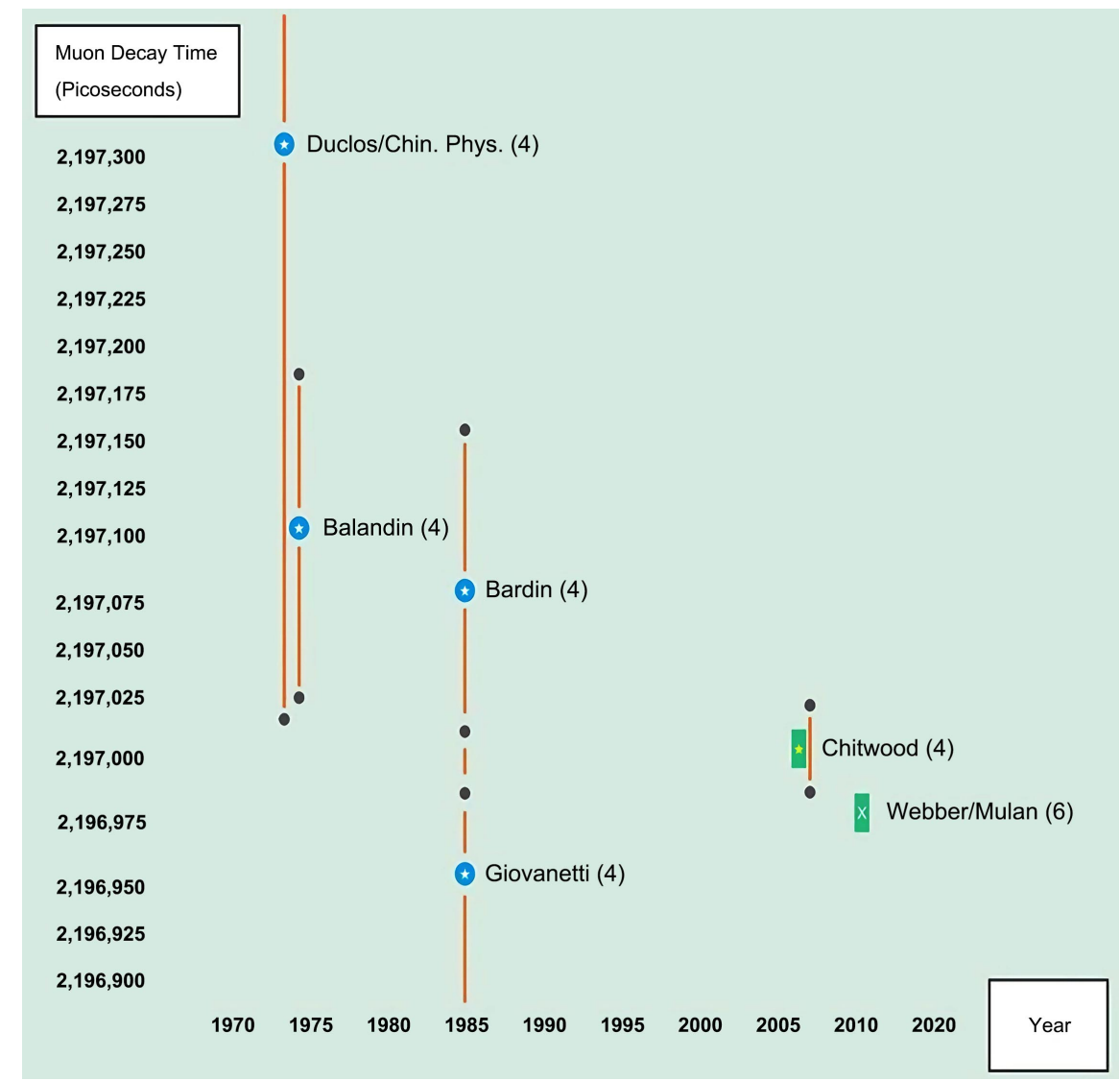

Figure 1. Data from Table 1 and Fig. 1a, page 63 of [1]).

outliers, with $P_{e}=2,330,000$ ps (we expect it to be exactly as measured most recently) and $P_{o}=2,080,000 \mathrm{ps}$ (most recent 2017 measurement) over the time interval of $t_{2}-t_{1}=2017-1946=71$ years, then the variation of the speed of time from Equation (1) would be

$$
(2080000-2330000) / 71=-3521 \mathrm{ps} / \text { year . }
$$

If the outliers are eliminated and only the more accurate MuLan data utilize, then $P_{e}=2,197,013$ ps [4] and $P_{o}=2,196,980.3 \mathrm{ps}$ [5] over the time interval of $t_{2}-t_{1}=2009.5-2007=2.5$ years, then the variation of the speed of time from Equation (1) would be

$$
(2196980.3-2197013) / 2.5=-13 \mathrm{ps} / \text { year . }
$$

In any event, the Table 1 exhibits most of the more accurate muon-decay times found and their estimated error. We recognize that the slowdown of clocks in ps per year, probably itself decreases or increases as time increases. Therefore, there may have been an actual "accelerated or decelerated slowdown" after the beginning of our Universe! However prior to this analysis, there was no a priori observational data of muon-decay time analyzed to indicate with certainty either a constant or a varying slowdown or speedup of the rate of time.

Under the supposition or working hypothesis that the aforementioned decrease in muon-decay time shortens as time increases, an interesting conjecture 
immerges: that the muon-decay process operates with a different "clock" or change in the speed of time, compared with the clock with which the rest of our Universe operates! Is there a possibility that muon decay has a clock that runs without variation at a fast or slower pace as time progresses? Under this assumption or working hypothesis the rate of slowdown of the time in our Universe is computed to be very roughly (not enough data to support a valid estimate of error) of between very approximately -13 ps per year (or $-4.1 \times 10^{-19} \mathrm{~s} / \mathrm{s}$ ) and -3500 ps per year (or $-1.1 \times 10^{-16} \mathrm{~s} / \mathrm{s}$ ) during the 71 year period between 1946 and 2017. Since we have no other muon-decay times to analyze, we will make the provisional assumption that the muon-decay rate of time change in our Universe does not remain a constant, but becomes smaller as the time in our Universe increases! There are $2.2 \times 10^{9}$ seconds in 71 years so the rate of the assumed rate of change is $\left([3500-13] \times 10^{-12}\right.$ seconds $) / 2.2 \times 10^{9}$ seconds $=-1.6 \times$ $10^{-19} \mathrm{~s} / \mathrm{s}$. Therefore in this case, with the 71 years centered about 1981, or approximately $4.32 \times 10^{17}$ seconds since the beginning of our Universe. These slowdowns per second over 71 years are very approximate and call for more Muondecay measurements having higher accuracy as well as more data on muon-decay-time found from other past times.

Figure 2 is a Notional plot of the change in the speed of time variation as a

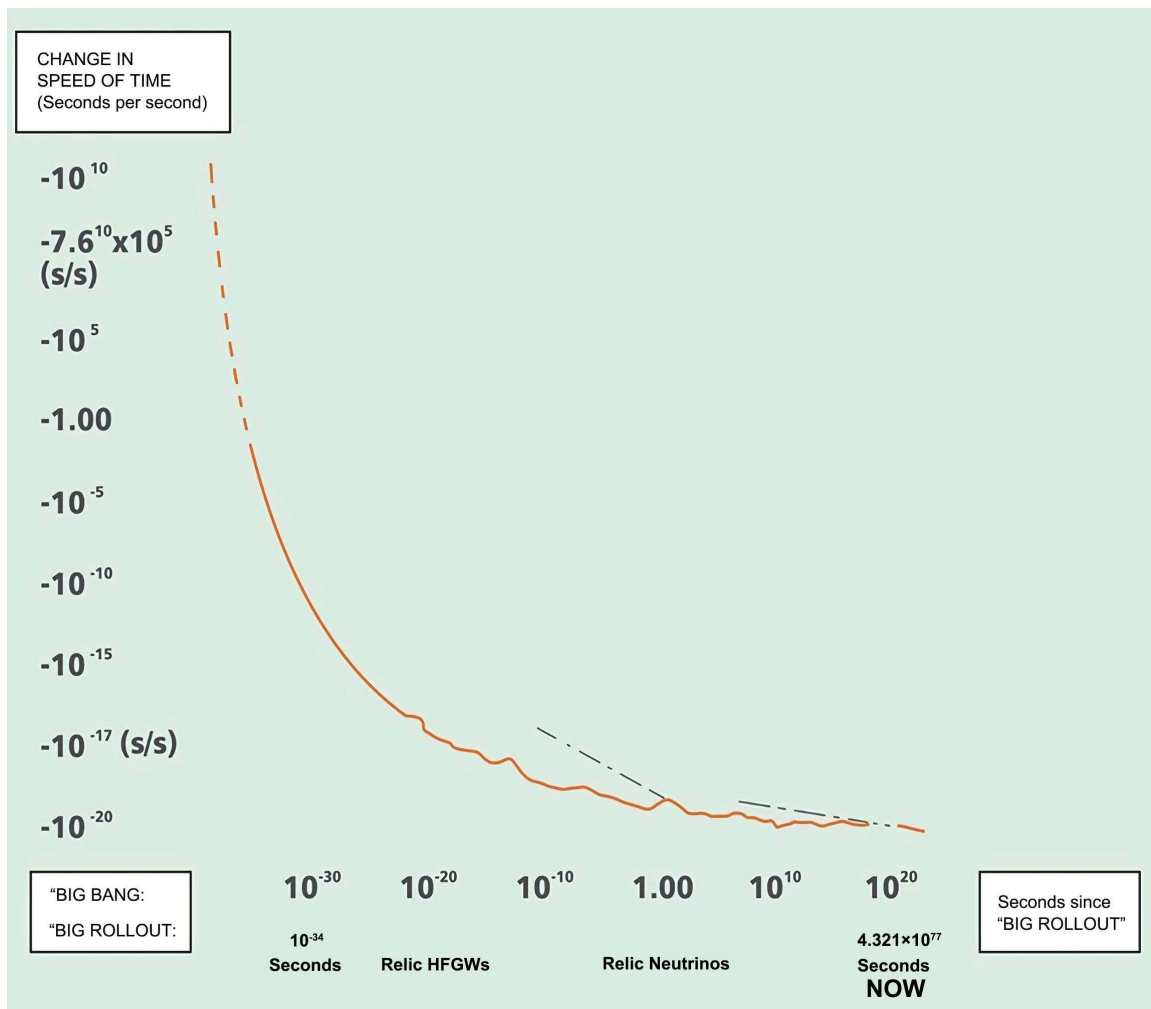

Figure 2. Notional graph from Fig. 3 of [11] of the change-of-speed-of-time variation with today's time dimension. The Figure is only a schematic and not intended for detailed analyses. Notice different slopes (tangents) and irregularities and the current time rate of about $10^{-17}$ seconds per second between $10^{-15}$ and $10^{-20}$ seconds per second shown by the expanded graduation scale on the ordinate near the "BIG BANG" or "BIG ROLLOUT". 
function of the time since the "Big Bang" or "Big Rollout" taken from Fig. 3 of [11]. It is only schematic and not intended for detailed analyses. The substantial increase in the speed of time value in the Notional and schematic graph of Figure 2, a nanosecond or less after the beginning of our Universe, is based upon our Rollout Theory of the beginning of our Universe and the high speed of time near the beginng of our Universe proposed by Baker [12]. The detection of High-Frequency Gravitational Waves (HFGWs) generated by processes occurring less than a nanosecond after the beginning of our Universe would provide the most important fundamental data for the formulation of a theory on the variation of the speed of time! The specific data points on the very approximate curve, quite close in time to our Universe's beginning would be disclosed by a study of the HFGWs emanating from the early Universe. Such a study could be obtained through utilization of the effect found by Li [13] and the Li-Baker HFGW Detector [14] as well as the analysis of the sensitivity and utilization of that Detector [15] [16] [17].

We assert that the Rollout Theory for our Universe [12] is simpler than some portions of the conventional Theory for the Big Bang: such as "...that the nascent Universe passed through a phase of exponential expansion soon after the Big Bang, driven by a positive vacuum energy density" (see Fig. 1 of [12]). Whereas the proposed Rollout Theory depends upon the simple concept that our Universe is similar to an ordinary clock or wristwatch that is slowing down as it ages, therefore by Occam's razor the Rollout Theory is preferable.

Although it would not affect the correctness of the theorized Theory of our Universe [12], more accurate measurements of muon decay time are needed in order to actually calculate an accurate local variation of the speed of time on Earth or indicate that muon decay time does not change with time and does not have its own "clock." The speed of time and/or the speed of time's variation may well depend upon "where" one measures the variation on the fabric of spacetime and/or the local mass distribution of matter or some other feature of our Universe! There may be a number of alternatives to this slowing-of-time analysis, but since time slowing in our Universe has some bearing on two other processes to be considered in this study, we will continue with it.

\section{Rotational Speed of a Portion of the Visible Disk of a Galaxy to Measure the Variation of the Speed of Time}

If the rate of time was greater in the past (these observations come from photons produced by galaxies millions or billions of years ago), then galaxies would appear to us today, with our slower clocks, to be rotating faster just as a watch in the past, if seen today, would appear to be moving its hands faster than our slower clocks as seen today as in Figure 3.

As discussed on pages 71-72 of [11] the galaxies do not rotate like a solid top. Rather the galactic stellar material rotates at different rates depending primarily upon their radial distance from the galaxy's center. In Figure 4 the grey dashed 
Galaxies APPEAR to rotate faster in the past if time was moving faster then. Astronomers have attributed this to a lot more mass or matter in them that holds them together so they can rotate fast and not pull apart. They call it "dark matter"

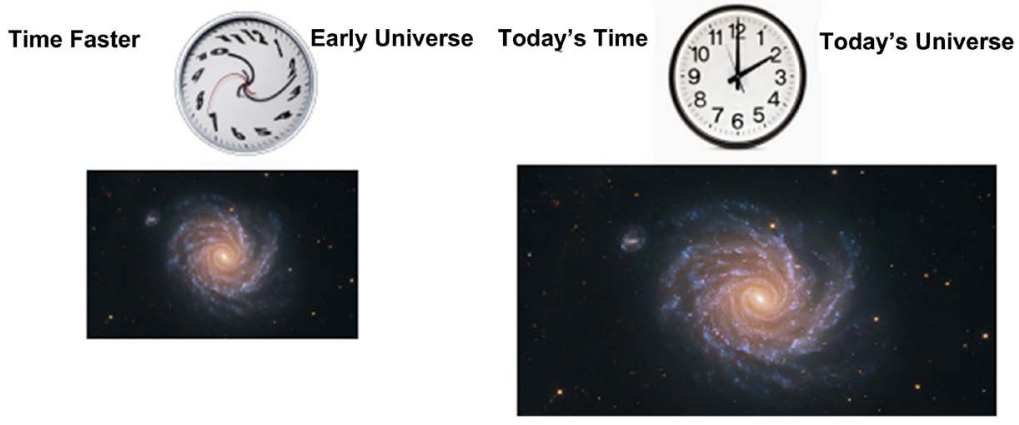

Figure 3. Rotational rate of galaxies.

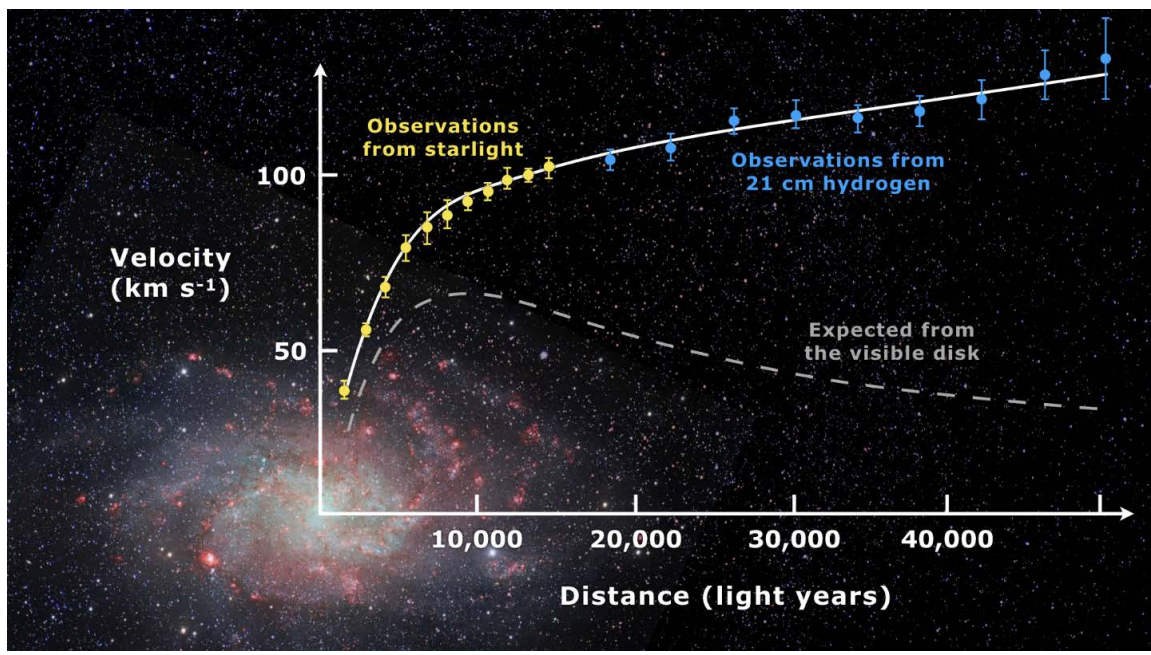

Figure 4. Typical galactic velocity curves. The upper-solid white-line curve is the observational data from galactic starlight (yellow data points) and radio-astronomy spectral analysis (blue data points) of the observed speed of galactic portions at various radial distances out from the galactic center of a typical galaxy such as Messier 33. The lower dashed-grey-line (speed) curves exhibit the expected speed, at the same radial distances, utilizing Astrodynamics [1] [2].

line exhibits the magnitude of the vector velocity in kilometers per second of stars in the galactic disk. It is obtained by Astrodynamic analyses [1] [2] of various galactic stars as a function of their radial distance in light years. The observed speed from the portion of the visible disk of a galaxy as measured from the Doppler Effect utilizing spectral data is shown by the solid white curve in Figure 4.

Consider the observational data for the galaxy, Messier 33, about 2.73 million light years away from the Earth as shown graphically in Figure 4. We will extract approximate values of the pertinent data from measurements of the drawing. This nearby galaxy is about the same distance from the beginning of our Universe as is the Earth. Consider a Doppler speed observation, $P_{o}$ of the luminous matter that shows the observed (by Doppler spectral analyses) tangential speed of "rotation" of a portion of the arms of the galaxy to be about $100 \mathrm{~km} / \mathrm{s}$ at about 
10,000 light years distant from the center of that galaxy. We will initially consider that distance from the galactic center for calculation since this observable part of a galaxy is moving at the maximum speed of galactic material approximately shown in Figure 4. The circumference of the assumed circular orbit of a galactic star (turns out to be a very poor assumption as will be discussed) at this 10,000 light year distance or $9.46 \times 10^{16} \mathrm{~km}$ radius, is $2 \pi \times 9.46 \times 10^{16} \mathrm{~km}=5.94 \times 10^{17}$ $\mathrm{km}$. Therefore as observed, this requires $5.94 \times 10^{17} \mathrm{~km} /(100 \mathrm{~km} / \mathrm{s})=5.94 \times 10^{15}$ seconds to complete one revolution or one orbital period of a star's circular orbit. The calculated and expected tangential speed, derived by applying conventional gravitational or Astrodynamic theory, is

$(65 / 100) \times 5.94 \times 10^{15}=3.86 \times 10^{15}$ seconds to complete one revolution or one orbital period. In order to compute Equation (1), we insert the difference between these two times and divide by the number of years for the photons to reach the Earth, $t_{1}-t_{2}=-2.73 \times 10^{6}$ years. Therefore the speed of time change at this position on the galaxy is $\left(5.94 \times 10^{15}-3.86 \times 10^{15}\right) / 2.73 \times 10^{6}=6.52 \times 10^{8}$ seconds per year! Compared to muon-decay computed speed of time on the Earth, this is extremely large. As will be emphasized later, such an enormous speed of time is based upon a completely erroneous, although interesting, assumption of a circular galactic orbit and will be discarded. Furthermore, from Figure 4, the speed of time would appear to increase in a very anomalistic fashion, even more so out nearer the periphery of the Messier 33 galaxy! As will be discussed, due to an increase in the speed of time in the past and miss-associated "background" stars measured, there may well be an over estimate of the speed of the galactic stars and especially miss association of these distant in spacetime apparently fast-moving stars with the galactic stars. Therefore in Figure 4, the upper solid line of observed Doppler-Effect observations could in actuality be much slower and closer, but might not overlap the lower estimated value of galactic rotational speed based upon conventional gravitational or Astrodynamic theory. The speed of stars beyond the 40,000 light-year radius are no doubt unrelated to Messier 33 and are observed from our Earth as having higher apparent speeds as our universe rolls out. In this case, the actual $21-\mathrm{cm}$ spectral-line-shift observational data of Figure 4 show those apparent stellar speeds due to the predicated slowdown of time from very high speeds, on average approach about 150 $\mathrm{km} / \mathrm{s}$ in the region of observable spacetime near Messier 33 and supports the new Rollout Theory [12].

To measure the rotation of a galaxy, observations must take into consideration the average shift of the spectrum of the galaxy. This will almost always result in a net spectral redshift for galactic stars, since it includes the expansion of the universe, and also our solar system's motion around the galaxy we are observing (the rotation of our planet, our orbit around the Sun, the Sun's motion around the galaxy, and the galaxy moving through the universe). The more rapidly the galaxy rotates the more the red shift. Distant stars in spacetime, further and older than a given galaxy, show a larger red shift the faster time is moving in their spacetime region. Therefore, if mistaken for galactic stars then an erroneous higher galactic 
rotational rate and "velocity curve" is mistakenly observed for the galaxy.

We must realize however, that the assumption of circular orbits for the observed stars is incorrect! We have no good information about how those orbits really are shaped. A far more fundamental concern is that "speed" is a scalar and not dependent on the trajectory of the speeding object. An analogy is that a 4-minute-mile Track Runner's speed at a given point is not measured by "distance per mile run per 240 seconds" or for a marathon runner not measured by "26.2 miles in so many seconds" or for a galactic star not measured by "single orbit distance per orbital period."! No, it is actually based upon the inverse of the time to move a given reference distance. In measuring the speed with which stars move in a galaxy it is for example the number of seconds to move a kilometer or a meter. Therefore, the $\mathrm{P}_{0}$ observed process time shown in Figure 4 is approximately $1 / 100 \mathrm{~km} /$ second or 0.01 seconds "per kilometer" and the $P_{e}$ expected process time also roughly measured from Figure 4 is approximately $1 / 64$ $\mathrm{km} /$ second or 0.0156 seconds "per kilometer". The expected process takes about 2.73 million years to reach the Earth, so $t 1-t_{2}=2.732 .73 \times 106$ years and Equation (1) is

$$
\begin{aligned}
& (0.010-0.0156) / 2.732 .73 \times 10^{6} \\
& =-2.08 \times 10^{-9} \text { seconds per year or }-2.080 \text { ps per year }
\end{aligned}
$$

A big difference from the orbital-period approach, but probably within the possible error of the relatively nearby Earth's muon decay speed-of-time determination of -13 ps/year to -3521 ps/year. Also this rate of time determined by spectral analyses is possibly underestimated because of time dilation plus gravitational potential!

As already emphasized, the significant departure of the observed speed of galactic portions from the expected speed in Figure 4 is important and supports the Rollout Theory of our Universe. The expected speed is based upon orbital analyses. The motion of the stars in a galaxy is considered to be an n-body problem discussed, for example, in Section 2.1 of (2). There exist no general analytical solutions for $n>2$, therefore General Perturbations do not apply and one must utilize Special Perturbations or numerical integration as discussed in Chapter 3 and Appendix D of (2). Presumably such techniques, including the effects of special and general relativity, GS and GR, were employed in the generation of the expected curve in Figure 4. The observed curve in Figure 4 involves the variation of the speed of time. We consider that curve at about a 40,000 light year radial distance. We discussed the rather startling departure of the observed speed and the "expected" speed for this very nearby Galaxy. Therefore, the P0 observed process time shown in Figure 4 for radial distances less than 30,000 light years is very approximately $1 / 120 \mathrm{~km} / \mathrm{second}$ or $0.000,008,3$ seconds "per meter" and the $P_{e}$ expected process time also roughly measured from Figure 4 is very approximately $1 / 40 \mathrm{~km} /$ second or roughly $0.000,025$ seconds "per meter". Note that in this case we use the standard meter not the kilometer for analysis! Therefore, from Equation (1) 


$$
\begin{aligned}
& (0.000,0083-0.000,025) / 2.73 \times 10^{6} \\
& =-6.12 \times 10^{-12} \text { seconds per year }=-6.12 \mathrm{ps} / \text { year } .
\end{aligned}
$$

But, of course, we should not jump to conclusions since the speed of the stars at the periphery of the Galaxy may include Doppler observations of those stars at a greater distance OBSERVED THROUGH the periphery of the Galaxy and operating in a higher-speed-of-time spacetime region of our Universe! However also recall that possibly the variation of the speed of time may also be dependent on the density of surrounding matter of the galaxy or other characteristics of the nearby features of spacetime. Like calculations based upon other observational data, this apparent increase the speed of time or cosmological effect (CE) must be taken into account in any comprehensive Theory developed for the change in the speed of time in our Universe. As has been pointed out, so far there is no a priori means to establish the speed of time. Let the observational data be our guide to a Theory of Time!

\section{Separation Speed of Celestial Objects to Measure the Speed of Time}

The Hubble Space Telescope (HST) observations of the stellar-object-separation speed of very distant supernovae showed that, a long time ago (billions of years ago), the universe was actually expanding more slowly than today. So the expansion of the universe apparently has not been slowing due to gravity, as it should! The expansion has apparently been accelerating! No one expected this since gravity should be slowing speeds down. No one knew how to explain the situation except to invent some invisible "dark energy" caused acceleration. So far no one has been able to detect this dark energy-truly a mystery! But wait! How is the speed of these very distant celestial objects' relative to our Earth measured? Again, like the speeds of portions of a galaxy, the speed is measured by the Doppler Effect!

According to our working hypothesis [12], the speed of time was greater in the past. Since we can only see stars as they were in the past, we suggest that speed of time was greater in the vicinity of those stars we observe and greater and greater the farther away they are (their photons taking longer to reach us). The situation is just like viewing a scene with a variable-rate movie projector. In an old movie projection suppose the film was moving faster through the movie projector than usual, like the time moving faster. The situation is that the people on the movie scene appeared to be moving fast, but their actual speed was the same, usual speed! In order to illustrate this point, let's consider another situation: From an observatory here, violin strings in a billions of light-year distant place with time running fast, would appear to vibrate faster and, if it were possible to hear the violin, then the violin's pitch would appear to be higher (like a spectrum showing a higher frequency and being more blue and less red). However, inside that billions of light-years away concert hall the violin strings would not appear to vibrate faster and violin's pitch would be unchanged! In fine, as we have just discussed, if time is running faster in a receding star's vicinity, then the reddening of stars will appear 
to be less since their spectra appears to move toward the bluer, higher frequency end and diminishes the observed Doppler-Effect-determined speed (as already noted, time dilation and gravitational potential have the opposite, i.e., cause a more reddening, effect). The situation would seem to a casual observer that the higher speed of time in the past would make the receding speed of celestial objects increased or seem faster. This is not the case, the receding speed appears decreased as measured with a Doppler-measurement due to increased speed of time! That is, due to an increased speed of time, the star's receding speed is actually larger than the spectral, Doppler-determined, receding speed shows! We will now explain in more detail this situation by the following story: A scientist sits in a train station and requests the station manager to tell him how fast the trains are moving when a receding train reaches a mile-away point. Like the recessional speeds of celestial objects, the scientist only considers the recessional speed of the trains. The first train to pass is going at a $30 \mathrm{mph}$, recessional speed at the mile-away point down the track. The scientist notes in his log book that the receding train's-whistles frequency drops a little from the whistle's normal frequency at the one-mile distance point. Of course this frequency drop seems reasonable, since the whistle's sound waves are stretched out a little as the train recedes. The second train to pass is moving at $60 \mathrm{mph}$ and the receding train's whistles frequency drops at the mile down-track point even more since the sound waves are stretched out even more by the rapidly receding train's whistle. The scientist records the whistle frequencies in his log of train-whistle frequencies for train receding at different speeds. He observes that receding train Whistle's frequencies drop more for faster receding trains since the sound waves are even more stretched out. The next day another train passes and the scientist wants to test out his work. The scientist tells the station manager that according to his log he expected that, from the frequency of the whistle, the currently receding train is going $30 \mathrm{mph}$. "No" says the station manager "...from my actual observations the train is moving at 60 mph mile at the down the track point from you." The scientist exclaims "But the sound waves are not stretched out as much and their frequency is not low enough for $60 \mathrm{mph}$ ". The station manager states that the actual pitch or frequency of this train's whistle had been changed by the Mechanics last night to a much higher frequency so the sound waves seem STRETCHED OUT like a 30 $\mathrm{mph}$ train! In the case of a receding stellar object, the increase in frequency is not accomplished by the Mechanics' whistle-increase modification, BUT BY THE INCREASED SPEED Of TIME INCREASING THE STELLAR OBJECTS APPARENT FREQUENCY! Or in the other by the story, like the violin sound's apparent increased frequency when heard from a distance.

The apparent increase in recessional speed (acceleration) between the Cosmic Microwave Background (CMB) very near the beginning of our Universe (at about 380,000 "years" after our Universes' beginning), of $6.75 \pm 0.05 \times 10^{4} \mathrm{~m} / \mathrm{s}$ per Mpc [18] and [19] to those of the Cepheid Variables (at about 163,000 light years distant, of $7.4 \pm 1.5 \times 10^{4} \mathrm{~m} / \mathrm{s}$ per Mpc (Table 5 of [18])) is simply due to the possibly high speed of time back at the time of the CMB. The Doppler-de- 
termined speed would be less than the true higher recessional CMB speed (producing a deceleration when compared to the Cepheid Variables speed) and agrees with a slowing due to gravity! No dark energy need be assumed!

The Hubble constant, $H_{0}$, is approximately $H_{0}=70[\mathrm{~km} / \mathrm{sec} / \mathrm{Mpc}]$. But can be expressed as the inverse of the time, $T$, in seconds for a celestial object to move an $\mathrm{Mpc}$ or $\left(3.09 \times 10^{22}[\mathrm{~m} / \mathrm{Mpc}]\right) /(70,000[\mathrm{~m} / \mathrm{sec}])=4.4 \times 10^{17}$ [seconds].

For calculations of the Process times, $T$, of celestial objects given their speeds of recession, we utilize the equation

$$
T=4.4 \times 10^{17} \times(S / 70) \text { (seconds) }
$$

where $S$ is the recessional speed of the celestial object in $[\mathrm{km} / \mathrm{sec} / \mathrm{Mpc}]$.

The CMB has a Speed, $S$ of $74[\mathrm{~km} / \mathrm{sec} / \mathrm{Mpc}]$. Therefore Time, $T=4.4 \times 10^{17} \times(74 / 70)=4.65 \times 10^{17}$ seconds for celestial objects, such as the CMB fairly near the beginning of our Universe, to separate a "given distance" of a Megaparsec. Under a "popular" Theory of our Universe (not however, proposed in [12]) that the separation speed of objects in our Universe should remain a constant, this number of seconds would be expected everywhere in our Universe, therefore $P_{e}=4.65 \times 10^{17}$ seconds.

Likewise for the more nearby Large Magellanic Cloud Cepheid's, which have an observed speed, $S$, of $67.5[\mathrm{~km} / \mathrm{sec} / \mathrm{Mpc}]$ Time,

$T=4.4 \times 10^{17} \times(67.5 / 70)=4.24 \times 10^{17}$ seconds $=P_{0}$. Of course, the reference or "given distance" is huge as is the time interval between observations of the separation speed of these celestial objects in our Universe. An alternative approach would be to define the "given distance" as simply the MKS meter as was utilized for the galactic measurements. Of course a kilometer could also have been chosen as the "given distance", so the choice is rather arbitrary. In the case of the galactic star's measurement, a galactic star's orbital period was not a valid, constant or unique "given distance", therefore the meter was selected as the reference or "given distance." In the case of the separation speed of celestial objects the Megaparsec in meters is a definitive unit of distance in MKS units and the same for all celestial objects under consideration. Also the assumption of a constant speed of recession is simply a popular concept and not involved in the working hypothesis Theory [12]. Therefore these calculations should be considered to be extremely provisional and needs to be examined very carefully!

Other "given distances" or "expected times" values for $P_{e}$ might in future be realized better utilizing, Fast Radio Bursts (FRBs), Soft Gamma ray Repeaters SGRs, pulsars, double star orbits, etc. If these measurements disclose that their frequency or periodicity increase slightly as they or their sources are measured to be further and further from our Earth, that is older and older, then their measurements might provide good, more detailed data on our Universes' variation of the speed of time.

From Equation (1), the variation of the speed of time between the CMB and the Large Magellanic Clouds, in which there are about 13 billion or $1.3 \times 10^{10}$ years between these observations or $t_{1}-t_{2}=1.3 \times 10^{10}$ years is: 


$$
\begin{aligned}
& {\left[P_{o}\left(t_{1}\right)-P_{e}\left(t_{2}\right)\right] /\left(t_{1}-t_{2}\right)} \\
& =\left[4.24 \times 10^{17} \text { seconds }-4.65 \times 10^{17} \text { seconds }\right] / 1.3 \times 10^{10} \text { years } \\
& =-3.15 \times 10^{6} \text { seconds per year }
\end{aligned}
$$

$=-3.15 \times 10^{18}$ ps per year or about $-0.1 \mathrm{~s} / \mathrm{s}$. Therefore over hundred trillion or more times larger compared to the muon-decay-time derived variation of the speed of time of -13 ps per year to -3500 ps per year values. This CMB value is extremely large and subject to considerable scrutiny but is still in keeping with our working hypothesis [12] that the speed of time was far greater in the distant past near the beginning of our Universe than today! Essentially both time and the space-dimension spacetime of our Universe commence expanding at the speed of light according to [12].

The CMB is close to the beginning of our Universe, nevertheless the CMB is not close enough to be particularly useful in developing a Theory for the variation of the speed of time. For that we require information from the HFGWs created at least a nanosecond nearer in time to the beginning of our Universe. What really happens at the "point" where time and space commence must await the analysis of the HFGW spectrum of the early Universe. But recall that the variation of the speed of time after that commencement "point" may well also depend upon "where" one measures the variation of time on the fabric of spacetime, the local mass distribution of matter or some other feature of our Universe!

\section{Muon-Decay Time Revisited and Non-Varying Rate of-Time (NVRT) Processes}

Is there something more fundamental going on concerning muon-decay time? Not just "muon decay operates with a different 'clock' or time than the clock the rest of us and our Universe uses." As discussed in Section 4 of [20], perhaps muon-decay time is a different kind of process. Let us explain the situation with another story: We will utilize the fictitious tale of a tribe called the "Muons" who originated billions of years ago near the beginning of time and exist even today. The Muons all have the unique capability to consistently run a mile in exactly four minutes. Recently a Muon runner came to my mile-long track. She asked if she could borrow my watch since she had misplaced hers. I agreed and handed over my watch with the admonition that my watch only showed the correct speed of time in my location at this specific local time. She looked at the watch and exclaimed: “...it is absolutely identical to the watch that I and my entire Muon tribe had used for billions of years ... my watches' rate of time is exactly the same, not too fast and not too slow, as the watch I had always had and lost!" If there is one thing these Muons know about, it is time!

The Muon runner ran my mile-long track and at the track's end, while looking at her "new" watch, she exclaimed "Perfect! My wristwatch shows exactly four minutes!" She told me that the Muons could not actually "see" the track - as a matter

of fact, they could not judge or "see" any distance! "We Muons cannot recognize or even comprehend the three dimensions of space-we only recognize the time di- 
mension." Also she stated that I should be careful using the wristwatch that I replaced the one I had given her. "Perhaps your replacement wristwatch was not perfect!" She said: "After looking at your replacement wristwatch I discovered it is flawed in that it seems to slow down with time, whereas the one you gave does not!"

What else does this story apply to? Let us suppose that, say, Nucleosynthesis is similar to muon decay and is a Non-Varying Rate of-Time (NVRT) Process and marches to the Nucleosynthesis own drum as it were. We make the very provisional assumption that there is no actual motion of the nuclei in space; that these high-energy collisions among nucleons only occur with a certain process-duration time just like muon decay! If we were able to observe this Nucleosynthesis process in operation today, then the process like muon decay would appear to take less and less time to be completed as our Universe's time slows down as measured by a Timer's stopwatch. Like the 4-minute-mile Muon runner, whose inherent "wristwatch time" seems moving faster than the current Universe time of a Timer. She stops her mile run before the Timer's stopwatch of today reaches the 4-minute point. Therefore, the Timer believes that she has run for a shorter time to complete the mile run (that is, to complete her "process")! In a sense we are observing compressed time from a vantage point of uncompressed time. So as we might observe Nucleosynthesis from afar through our telescopes today, the process would appear to occur more and more quickly over the years of observation just like the process of muon decay! If that does not occur, then Nucleosynthesis is not a NVRT process otherwise the process is a NVRT process!

There may be other transient processes or subsystems that involve one or more quantum-mechanical sub-reactions, some well understood and some not well understood, that in total comprise a complete, possibly multiple-step process having a well-defined beginning and end. This is the proposition:

Proposition (page 65 of [11]) that some complex processes or sub systems are "marching" to their own intrinsic" time" or timeframe that is independent of the flow of "time" in our Universe. (We call them Non-Varying Rate of-Time (NVRT) Processes.)

That is, besides muon decay there may be other such process that we define as the Non-Varying Rate of-Time (NVRT) Processes. Such processes do not "go with flow" of time slowing in our Universe. Such NVRT processes, according to our working hypothesis, may include those that generate Big-Bang Nucleosynthesis (BBNs) generation of Oh My God (OMG) very high-speed particles, Fast Radio Bursts (FRBs), Soft Gamma ray Repeaters SGRs (the latter two possibly from Magnetars) and perhaps weak nuclear reactions of proton-proton chain (affecting stellar luminosity but far more likely not to be NVRT processes since they probably are "space-coordinate" dependent in their operation). We will concentrate the following analyses on muon decay since we have studied that process in some detail. By the way, galactic motion, black-hole mergers, Nova and other more extensive in motion in the three space coordinates and less quantum-mechanical in operation are not NVRT processes. Unlike the hypothetical Muon runners they recognize the three space dimensions. Also their time varies as time 
mainly does in our Universe-they "go with the flow!"

It is important to understand that the Non-Varying Rate of-Time (NVRT) Processes working hypothesis or concept is not directly related to the Rollout of the Universe Theory [12], multiuniverses, special or general relativity, hyperspace, parallel universes, etc. the NVRT process is a very new and different concept!

Let us continue the discussion by using a standard muon-decay illustration as shown in Figure 5.

The very most important property of this standard diagram of muon decay

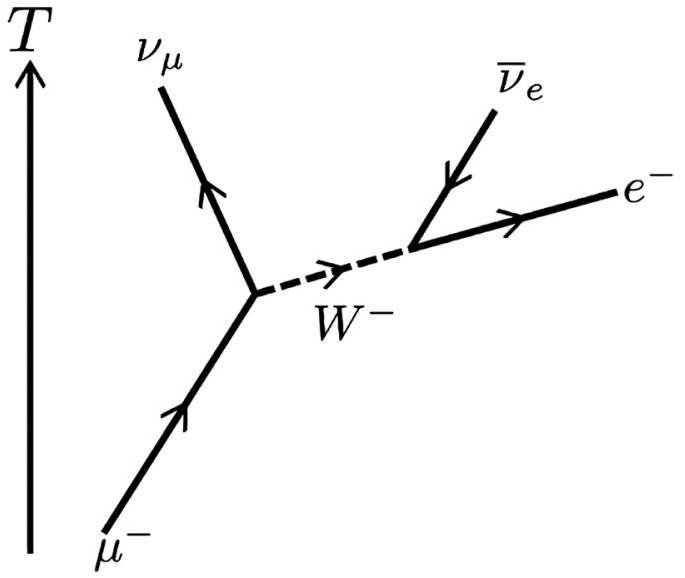

Figure 5. Standard diagram of muon decay.

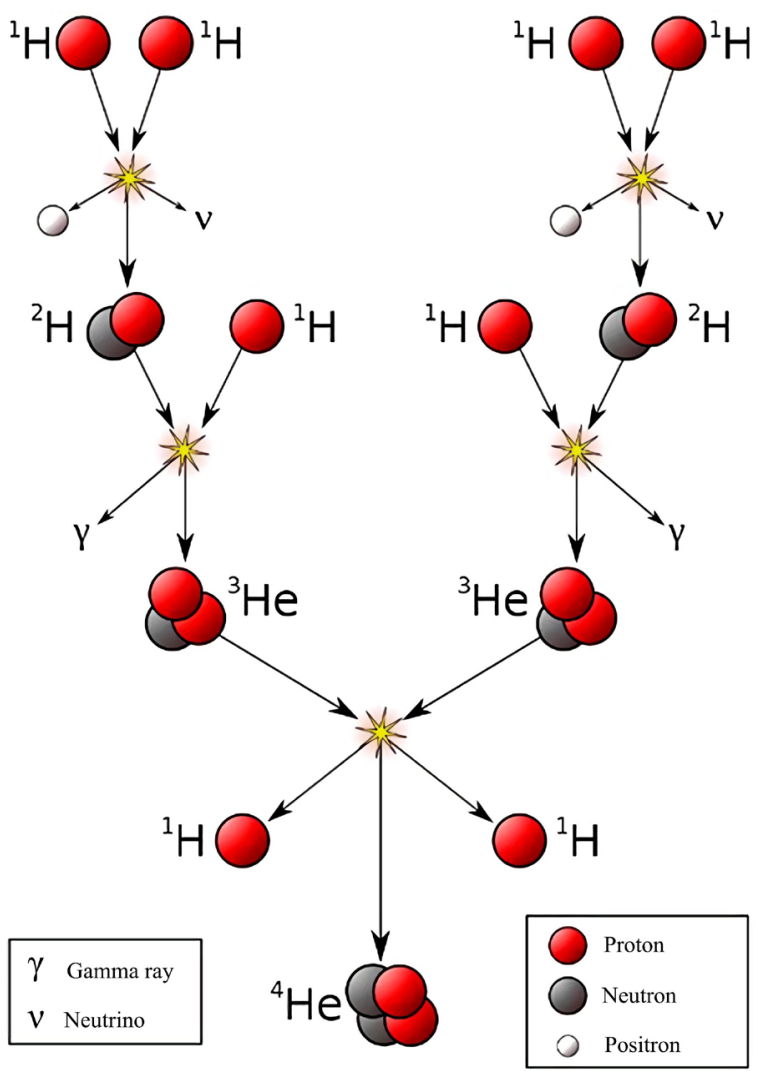

Figure 6. Standard diagram of proton-proton chain reaction. 
shown in Figure 5, is that there is only one dimension involved: $\mathrm{T}$ or time. No space dimensions at all! We contrast this with the standard diagram of proton-proton chain reaction, which generates stellar luminosity, shown in Figure 6.

In this case an "alarm clock" that signals the beginning (when the alarm clock is set off by an experience e.g., a collision with a cosmic ray) and simply signals the end of the muon decay process when the alarm clock "rings"! Hydrogen and helium atoms move and physically collide with each other. They actually move through the dimensions of space! The idea is that NVRT processes, such as muon decay, are in a sense not actually a part of the spacetime continuum! Specifically, they have their own clock, that alarm clock time interval is completely independent of where in space the collision occurs or especially any "space change" in the Muon's decay process during that process!

Do the Non-Variable Time Rate (NVRT) processes, like muon decay processes, relate to processes such as production of new elements and those that are involved in the mysterious core-collapse of supernova [21] happening billions of years ago? If such is the case, then we may have another mysteries solved!

\section{Conclusions}

An equation is presented involving the observed time for a process to be completed and the expected time for the process to be completed. The difference between these two times is attributed to a change in the speed of time. For the Process of muon decay the speed of time is found to decrease at the rate of between -13 picoseconds, ps, per year and -3521 ps/year at about the date of 1981 on Earth. Although it would not affect the correctness of the theorized Theory of our Universe [12], more accurate measurements of muon decay time are needed in order to actually calculate an accurate local speed of time on Earth or indicate that muon decay time does not change with time and is not a NVRT process. For a galaxy, such as Messier 33, the variation of the speed of time appears there to be $-6.12 \mathrm{ps} /$ year to at most $-2080 \mathrm{ps} /$ year (at the galaxies' outskirts) as a very provisional determination. However this speed of time for Messier 33 may actually be caused, at least in part, by the Doppler shift of stars observed beyond the Galaxy in spacetime regions of higher speed of time and apparent higher speed or some other effect. However, no dark matter need be assumed. From the speed of separation of celestial object as our Universe progresses, we find that in the time between the observations of the receding speeds of the CMB and the Large Magellanic Cloud Cepheid's of approximately thirteen billion year, there is a speed of time change of $-3.15 \times 10^{18}$ ps per year or about $-0.1 \mathrm{~s} / \mathrm{s}$. This calculation is in keeping with the theorized very much higher speed of time in the past of the CMB near the beginning of our Universe, predicted by [12] and the much slower speed of time in our current observations of the relatively nearby (in time and distance) Large Magellanic Cloud Cepheid speed. There is no acceleration of the speeds of these celestial objects speed of separation. Those separation speeds 
are decreasing as usually predicted by gravity and by using our speed-of-time theory, so that dark energy is not required!

Other determinations of the variation of the speed of time, and independent of special and general relativistic effects, might be by utilizing the Processes involving Fast Radio Bursts (FRBs), Soft Gamma ray Repeaters (SGRs), pulsars, double star orbital periods, etc.; of course only if these measurements are precise enough to disclose that their frequencies or rates appear to increase slightly as they or their sources are measured to be further and further from our Earth.

Other possible indicators of the variation in the speed of time besides muon decay time, quite valuable because they are independent of special and general relativity effects, might be found in meteoritic composition change over hundreds of thousands or millions of years. The research by Turner, et al. [22] found time differences in meteoritic-composition analyses that might relate to the speed of time: They found "... that the fluid-mobile uranium ion $\mathrm{U}^{64}$ moved within the past few 100,000 years ... This time scale is less than the cosmic-ray exposure age... when they were ejected into space. Fluid flow occurred after melting of ice" by impact heating (ablation) or solar heating. Or possibly, the effect was the result of the change in the speed of time right after the Earth was formed and today. The process here is the melting of the ice, the time it would be expected to occur and the time when it actually occurred.

With regard to galactic data to be utilized to compute the speed of time; in Section 3 we have computed the difference between the velocity curves in Figure 4 of the nearby Messier 33 galaxy would lead to a speed of time between about -2 and $-2,000$ ps/year. However, galaxies closer to the beginning of our Universe might also lead to estimates of the speed of time. As summarized by Wardlow [23] "... key features of a mature galaxy arose more rapidly than has been thought." Lelli, et al. [24] state "We conclude that massive bulges and regularly rotating disks can form more rapidly in the early Universe than predicted by of galaxy formation." Therefore the speed of time may be roughly computed by differencing the expected time and the observed time that features of galaxy formation appear.

We concluded with a study of Processes like muon decay, which may operate with a "different clock," a clock that does not participate in the variation in the speed of time that the rest of our Universe does-we call these Processes NonVarying Rate of Time or NVRT processes.

The speed of time and/or the speed of time's variation may well depend upon "where" one measures the variation on the fabric of spacetime and/or the local mass distribution of matter or some other feature of our Universe.

In order to establish a Theory for the origin and variation of the speed of time, we conclude that HFGW detection is required to understand the activity of Processes at a nanosecond or less after the beginning of our Universe [16]. We contend that primordial or relic HFGWs were propagated before our Universe became transparent to electromagnetic radiation. If such primordial HFGWs 
can be detected by the Li-Baker HFGW detector, discussed in connection with our analyses of Muon decay, then their observations may not only contain information on the speed of time, but information, gained by means of the analyses of their frequency spectrum, concerning the processes themselves.

\section{Acknowledgements}

Andy Beckwith, Nagib Callaos, Paul Murad, R. Clive Woods, Eric Davis, Gary Stephenson and Giorgio Fontana have provided encouragement for many of our "out of the box" ideas including our theory of the variable speed of time. Mounir Belgacem has given valuable assistance with the preparation of the figures.

\section{Funding}

All funding for this paper was exclusively and personally provided by the authors and no external funding was utilized.

\section{Authors' Contributions}

Conceptualization and all analyses: R.M L B., Jr and B. S. B. and introduction of entropy and many HFGW-detection concepts FY L.

\section{Conflicts of Interest}

There were no competing interests of any author.

\section{References}

[1] Herrick, S. (1971) Astrodynamics: Orbit Determination, Space Navigation, Celestial Mechanics, Volumes 1 and 2. Van Nostrand, Princeton.

[2] Baker Jr., R.M.L. (1967) Astrodynamics: Applications and Advanced Topics. Academic Press, New York.

[3] Conversi, M., Piccioni, O. and Pancini, E.P. (1946) Pancini, Piccioni (CPP) Experiment. Slide 10.

http://www0.mi.infn.it/ neri/HomePage/Teaching files/Esperimento CPP.pdf

[4] Olive, K.A. (2014) Chinese Physics C, 38, $648 \mathrm{ff}$. https://doi.org/10.1088/1674-1137/38/9/090001

[5] Webber, D.M. (2011) Physical Review Letters, 106, Article ID: 041803. https://doi.org/10.1103/PhysRevLett.106.041803

[6] Lindy, R.A. (1962) Physical Review, 125, 1686-1696. https://doi.org/10.1103/PhysRev.125.1686

[7] Tischchenko, V. (2013) Nuclear Physics B-Proceedings Supplements, 225-227, 232 235. https://doi.org/10.1016/j.nuclphysbps.2012.02.048

[8] Barazandeh, C. (2016) Journal Physics Conference Series, 770, Article ID: 012050. https://doi.org/10.1088/1742-6596/770/1/012050

[9] Physics OpenLab. (2016) Cosmic Ray Meeting, February, 2017, Slide 10: $2.050 \pm$ $0.040 \mu$ sec, Slide 11: $2.080 \pm 0.110 \mu \mathrm{sec}$, Slide 12: $1.90 \pm 0.190 \mu \mathrm{sec}$ (2017). http://physicsopenlab.org/2016/01/10/cosmic-muons-decay

[10] Adams, M. (2017) Cosmic Ray Meeting. February, 2017, Slide 10, Slide 11, Slide 12. 
https://indico.cern.ch/event/596002/contributions/2463437/attachments/1410577/2 157296/Adams-Rome.pdf

[11] Baker Jr., R.M.L. (2019) Journal of Space Science and Technology, 25, 60-77. https://doi.org/10.15407/knit2019.03.060

[12] Baker Jr., R.M.L. (2020) Journal of High Energy Physics, Gravitation and Cosmology, 6, 1-16.

[13] Li, F. and Baker Jr., R.M.L. (2007) International Journal of Modern Physics B, 21, 3274-3278. https://doi.org/10.1142/S0217979207044366

[14] Baker Jr., R.M.L. (2001) Gravitational Wave Detector. Chinese Patent No. 018144223.0.

[15] Clive Woods, R., Baker Jr., R.M.L., Li, F., Stephenson, G., Davis, E. and Beckwith, A.W. (2011) Journal of Modern Physics, 2, 498-518. https://doi.org/10.4236/jmp.2011.26060

[16] Beckwith, A.W. and Baker Jr., R.M.L. (2019) Journal of High Energy Physics, Gravitation and Cosmology, 6, 103-122.

[17] Li, F.-Y., Wen, H., Fang, Z.-Y., Li, D. and Zhang, T.-J. (2020) The European Physical Journal C, 80, 879. https://doi.org/10.1140/epic/s10052-020-08429-2

[18] Aghanim, N., Akrami, Y., Ashdown, M., Aumont, J., Baccigalupi, C., Ballardini, M., Banday, A.J., Barreiro, R.B., Bartolo, N., Basak, S., Benabed, K., Bernard, J.P., Bersanelli, M., Bielewicz, P., Bock, J.J., Bond, J.R., Borrill, J., Bouchet, F.R., Boulanger, F., Bucher, M., Burigana, C., Butler, R.C., Calabrese, E., Cardoso, J.F., Carron, J., Casaponsa, B., Challinor, A., Chiang, H.C., Colombo, L.P.L., Combet, C., Crill, B.P., Cuttaia, F., De Bernardis, P., De Rosa, A., De Zotti, G., Delabrouille, J., Delouis, J.M. and Di Valentino (2020) Astronomy \& Astrophysics, 641, 56. https://doi.org/10.1051/0004-6361/201936386

[19] Riess, A.G., Stefano, C., et al. (2019) The Astrophysical Journal, 876, 217-243. https://doi.org/10.3847/1538-4357/ab1422

[20] Baker Jr., R.M.L. and Baker, B.S. (2021) Journal of Systematics, Cybernetics and Informatics Special Issue "Rigor and Inter-Disciplinary Communication", 18, 217 243.

[21] Burrows, A. and Vartanyan, D. (2021) Nature, 589, 29-39. https://doi.org/10.1038/s41586-020-03059-w

[22] Turner, S., McGee, L., Humayan, M., Creech, J. and Zanjda, B. (2021) Nature, 371, 164-167. https://doi.org/10.1126/science.abc8116

[23] Wardlow, J. (2021) Science, 371, 674-675. https://doi.org/10.1126/science.abg2907

[24] Lelli, F., Di Teodoro, E.M., Fraternali, F., Man, A.W.S., Zhang, Z.-Y., De Breuck, C., Davis, T.A. and Malolino, R. (2021) Science, 371, 713-716. https://doi.org/10.1126/science.abc1893 